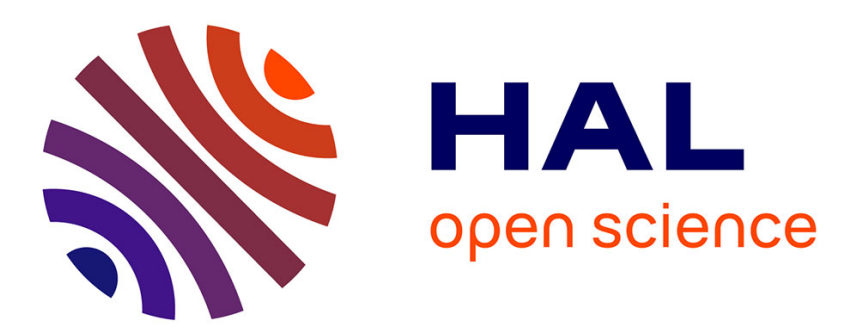

\title{
Enjeux et limites d'une amelioration des pratiques logistiques dans les hôpitaux publics français
}

\author{
Nathalie Sampieri-Teissier
}

\section{To cite this version:}

Nathalie Sampieri-Teissier. Enjeux et limites d'une amelioration des pratiques logistiques dans les hôpitaux publics français. Logistique \& Management, 2004, Spécial Logistique Hospitalière, pp.31-39. hal-01237328

\section{HAL Id: hal-01237328 \\ https://hal.science/hal-01237328}

Submitted on 3 Dec 2015

HAL is a multi-disciplinary open access archive for the deposit and dissemination of scientific research documents, whether they are published or not. The documents may come from teaching and research institutions in France or abroad, or from public or private research centers.
L'archive ouverte pluridisciplinaire HAL, est destinée au dépôt et à la diffusion de documents scientifiques de niveau recherche, publiés ou non, émanant des établissements d'enseignement et de recherche français ou étrangers, des laboratoires publics ou privés. 


\title{
Enjeux et limites d'une amélioration des pratiques logistiques dans les hôpitaux publics français
}

\author{
Nathalie SAMPIERI-TEISSIER \\ Maître de Conférences, IUT d'Aix en Provence, Université de la Méditerranée, CRET-LOG \\ sampieri.nathalie@wanadoo.fr
}

Les organisations sanitaires, plus particulièrement les hôpitaux publics, cherchent, sous l'impulsion des récentes réformes, à dégager de nouvelles sources de productivité, et à améliorer leur qualité. Les processus organisationnels deviennent ainsi l'objet de nombreuses réflexions collectives et transversales et la logistique n'échappe pas à cet engouement managérial. Ainsi constate-t-on, depuis une dizaine d'années environ en France, un accroissement des démarches d'amélioration autour de cette fonction dans les hôpitaux (voir par exemple CHU de Montpellier, Hôpital Européen Georges Pompidou,...). On évoque même la possibilité de mettre en œuvre un " health care supply chain management ». Derrière l'enjeu indéniable de ces démarches, qui traduisent des niveaux de développement divers d'un établissement à l'autre, se cachent toutefois des limites dont il faut prendre conscience, afin de contextualiser ces outils, dont l'origine est avant tout celle de l'industrie, de la grande distribution et des prestataires logistiques.

Les démarches managériales portant sur le processus logistique connaissent des développements importants dans les établissements hospitaliers de la plupart des pays occidentaux [Landry et Beaulieu (1)]. Elles demeurent toutefois encore émergentes en France, bien que de plus en plus prégnantes dans les projets d'établissement. En effet, les organisations sanitaires, plus particulièrement les hôpitaux publics, cherchent, sous l'impulsion des récentes réformes (loi du 31 juillet 1991, ordonnances du 24 avril 1996), à dégager de nouvelles sources de productivité, et à améliorer leur qualité. Les processus organisationnels deviennent ainsi l'objet de nombreuses réflexions collectives et transversales et la logistique n'échappe pas à cet engouement managérial. Ainsi constate-t-on depuis environ une dizaine d'années un accroissement des démarches d'amélioration autour de cette fonction dans les hôpitaux (voir par exemple CHU de Montpellier, Hôpital Européen Georges Pompidou,...).

Toutefois, ces bouleversements organisationnels adoptent souvent une vision tronquée de la logistique, soit par une limitation du champ d'action à des missions d'intendance (restauration, blanchisserie...), soit par une restriction du pilotage aux flux dits « classiques». En effet, elles excluent la plupart du temps les flux de patients. Or, la spécificité des activités de service engendre la nécessité de distinguer la logistique " classique » et la logistique « de service» [Mathe (2)].

Cette approche duale de la logistique laisse apparaître des solutions organisationnelles diverses, émergeant des établissements et de 
leurs partenaires, ou encore de courants théoriques des sciences de gestion. Les enjeux de ces changements locaux sont très importants à la fois dans une perspective financière (par exemple, réduction des stocks, diminution des durées de séjour), humaine (les soignants pouvant se recentrer sur leur cœur de métier), ou « commerciale » (réduction des temps d'attente des patients, amélioration de la qualité des soins...).

Cependant, il est important de considérer les limites de certaines démarches logistiques, notamment celles qui visent à intégrer l'ensemble des partenaires internes et externes dans le processus de pilotage. En effet, il est nécessaire de prendre en compte le contexte spécifique de l'hôpital en général, et de chaque hôpital en particulier, comme par exemple l'indépendance des médecins au sein de la structure ou encore l'appartenance à des réseaux de soins, qui peuvent contraindre fortement les solutions organisationnelles envisagées par un établissement.

Cet article s'attachera à présenter les enjeux d'une approche intégrée de la logistique, tant pour les flux traditionnels que pour les flux de patients. Pour cela, il se basera notamment sur les résultats d'une étude empirique mettant en évidence une typologie des pratiques logistiques dans les établissements publics hospitaliers français [Sampieri (3)]. La présentation de ces résultats sera résolument synthétique dans la mesure où un article y est entièrement consacré dans un numéro précédent de la revue Logistique \& Management [Sampieri-Teissier (28)]. Puis, il s'agira d'exposer les limites des démarches intégratives et des solutions présentées comme « idéales » par certains établissements.

\section{Les enjeux d'une approche intégrée de la logistique ... les origines industrielles du concept de logistique intégrée}

La logistique apparaît seulement dans les années 1950-1960 dans les entreprises industrielles; elle s'intéresse alors essentiellement aux questions de transport et d'entreposage, qu'elle cherche à optimiser. Elle ne se développe réellement en France qu'à partir de 1965 [Colin et Pache (4)]. Elle est, à l'époque, considérée comme "le transport envisagé dans la perspective de la fonction distribution du chargeur » [David (5)]. Elle reste limitée au traitement administratif et physique des produits finis, c'est-à-dire depuis la sortie de la production jusqu'au consommateur final. Ce n'est que dans les années 1970 que l'on prend conscience de l'importance d'une coordination des flux physiques de l'aval vers l'amont, avec les travaux d'Heskett (6).Depuis lors, la logistique ne va cesser d'évoluer en exploitant cette idée de flux tirés par l'aval, qui est fortement liée au concept d'intégration. En effet, faire remonter l'information depuis le consommateur jusqu'aux sources mêmes d'approvisionnement, repose nécessairement sur une forte intégration de la gestion des flux à l'ensemble des fonctions de la firme, dans un premier temps, puis aux différents partenaires de l'entreprise (on parle ainsi de Supply Chain Management). C'est par ce biais, que la logistique peut jouer son rôle de médiateur aux interfaces, aidant ainsi à une meilleure coordination des flux physiques. Son étendue transversale, intra et inter-organisationnelle, implique une démarche anticipatrice et proactive qui nécessite de prendre en compte le pilotage des flux au plus tôt, afin d'assurer leur fluidité et leur continuité, et donc de limiter les stocks et les goulets d'étranglement.

Il est important de préciser que les évolutions de la gestion des flux sont longues à mettre en œuvre. De plus, force est de constater qu'il existe des disparités importantes en terme de maturité logistique, non seulement d'une entreprise à l'autre, mais aussi d'un secteur à l'autre [Colin (7)].

Cette situation peut également s'observer au niveau des établissements hospitaliers français. Même si les enjeux d'une plus grande intégration logistique sont clairement perçus par les acteurs, le secteur de la santé connaît un retard très important en la matière, notamment au niveau des points d'utilisation des ressources, c'est-à-dire des services de soins (Cf. paragraphe sur la typologie). Il convient donc, avant tout, de s'interroger sur la définition même de la logistique hospitalière et sur ses enjeux.

\section{... appliqué au contexte hospitalier : définition et enjeux d'une intégration de la logistique.}

Un nombre croissant de démarches de réflexion autour du thème de la logistique dans les hôpitaux français [voir par exemple Caillet (8), Cros et Guillaume (9), ou encore Moujart (10)], se développent notamment à l'initiative des pharmaciens hospitaliers (11). On note également l'adaptation de concepts, encore récents dans l'industrie, au milieu hos- 
pitalier. Certains professionnels font ainsi appel à des termes tels que le supply chain management, l'effective customer response ou encore la gestion partagée des approvisionnements [Bisch (12)]. Ceci témoigne en partie d'une prise de conscience collective du rôle que peut jouer le management logistique dans les établissements hospitaliers.

Toutefois, malgré cet intérêt croissant pour la gestion des flux dans les établissements hospitaliers et les enjeux incontestables qu'elle revêt [les coûts logistiques représentent, selon les pays et les études, de 30 à $40 \%$ des coûts des établissements hospitaliers, Landry et Beaulieu (13)], la logistique demeure encore trop souvent cantonnée sur le terrain, à des missions d'intendance telles que la restauration ou la blanchisserie ( 9 et 14). Cette vision tronquée de la logistique émerge également au travers du manuel d'accréditation ${ }^{1}$ publié en 2000 par l'ANAES'. Ce constat est par ailleurs confirmé par une enquête exploratoire qui a été conduite sur trois sites hospitaliers en 1999 (3). Ainsi, les hôpitaux publics français se situent essentiellement au stade premier de la typologie décrite par Landry et Beaulieu (1, p.101). En effet, les établissements essaient avant tout de minimiser les impacts négatifs provenant des activités logistiques.

En outre, la représentation que les acteurs se font de la logistique se limite au pilotage des flux physiques « classiques ». Elles excluent la plupart du temps les flux de patient. Or, la spécificité des activités de service engendre une distinction entre la logistique « classique » et la logistique « de service ».

La logistique " classique », ou traditionnelle, s'attache à la gestion des matières premières servant directement ou indirectement à la production du service : médicaments, autres matériels médicaux, biens de restauration, produits ménagers, linge... Cette approche correspond au champ d'analyse étudié par les chercheurs du groupe Chaîne (par exemple 1). Ces auteurs n'excluent pas pour autant l'enjeu que revêt le second volet de la logistique hospitalière.

La logistique « de service » (de régulation de la production ou logistique de réaction) consiste en la gestion des flux de patients, en agissant simultanément sur la demande et les capacités. Son objectif est d'arbitrer entre le temps d'attente des patients et l'optimisation des capacités.

Adopter une vision élargie de la gestion des flux, tant au niveau de l'intégration de ces der- niers, qu'au niveau du type même de flux (traditionnels et de service), permet de mettre en perspective des enjeux considérables pour les organisations sanitaires, pour les patients, et pour la collectivité dans son ensemble.

Tout d'abord, concernant les flux physiques traditionnels, rappelons les enjeux d'une intégration de la gestion des flux [Sampieri (15)]. Cette dernière pourrait tout d'abord permettre, à terme, une baisse notable des coûts liés à un processus logistique défaillant, c'est-à-dire des surcoûts de stockage (évalués par exemple à 3 mois de stock pour l'hôpital Laennec à Paris- Ducasse, 1995), la multiplication des lieux de stockage (encombrement, risque de perte...) ou encore une mauvaise qualité du stock (péremption, ruptures...). Elle pourrait également contribuer à un gain de temps notable pour les soignants, qui seraient déchargés des tâches logistiques pour se recentrer sur leur cœur de métier, qui est le soin aux patients (on évalue actuellement le temps consacré aux activités logistiques à $10 \%$ du temps du personnel soignant). En outre, la recherche de coordination entre les différents services, logistiques et de soins, pourrait également conduire à une amélioration du dialogue et par ce biais à un décloisonnement organisationnel.

Si l'on élargit la logistique à l'ensemble des acteurs de la chaîne (fournisseurs, prestataires logistiques...), les enjeux sont d'autant plus importants, puisqu'une gestion intégrée devrait contribuer à une réduction supplémentaire des coûts, mais aussi à une amélioration de la qualité du processus logistique (car ce sont des professionnels qui s'en chargent, qui en outre, bénéficient de leur expérience acquise sur divers hôpitaux et cliniques) et à une augmentation notable de la qualité des soins [l'hôpital pouvant se recentrer sur son cœur de métier - Prahalad et Hamel (16)].

Pour résumer, une intégration intra et inter-organisationnelle de la logistique traditionnelle pourrait conduire non seulement à une amélioration de l'efficience des organisations hospitalières (des gains de productivité), et de la qualité du processus logistique (moins de ruptures de stocks, de produits périmés...), mais aussi à un gain de temps important pour les soignants, qui pourraient se consacrer plus amplement à leurs patients, et accroître ainsi la qualité des soins.

S'agissant d'une intégration de la logistique de service (les flux de patients), les enjeux sont comparables, quoique différents. Elle vise avant tout l'amélioration de l'articulation
1 - C'est l'équivalent pour les hôpitaux de la certification de type ISO 9000. Le manuel est disponible sur le site Internet de l'ANAES www.anaes. $f$ r.

2 - Agence Nationale d'Accréditation et d'Evaluation en Santé. 
entre la demande (émanant des patients) et les capacités (ressources humaines et matérielles). Diverses solutions peuvent être avancées : mise au point d'un système de réservation, programmation journalière de l'utilisation des capacités (par exemple, pour les appareils d'imagerie médicale), simplification des formalités d'admission, anticipation des examens à réaliser en fonction de la pathologie du patient... Elles permettraient des bénéfices importants pour le patient qui verrait, d'une part, se réduire l'ensemble de ses déplacements, en nombre et en distance, et d'autre part, s'améliorer l'information visant à l'aider dans son orientation (signalisation adaptée aux trajectoires et besoins des patients), donc, par voie de conséquence, une réduction de la durée globale de séjour. Cet élément bénéficierait directement à l'hôpital, et à l'ensemble de la collectivité. En outre, les organisations hospitalières réaliseraient des gains de productivité substantiels en améliorant la gestion de leurs ressources qui sont, pour beaucoup, très coûteuses (l'utilisation du plateau technique en est un bon exemple) ${ }^{3}$.

En définitive, l'intégration de la logistique, tant au niveau des flux traditionnels que des flux de patients, revêt des enjeux particulièrement importants pour accroître l'efficience des hôpitaux, la qualité des soins prodigués, et donc la satisfaction des patients et de la collectivité.

La prise en compte de ces enjeux se traduit, sur le terrain, par l'adoption de solutions organisationnelles diverses, essentiellement centrées sur les flux traditionnels. En effet, la réflexion logistique peut aller d'actions limitées par exemple à la fonction achat ou à la gestion des stocks [Hôpital principal de Dakar, Gottsmann (17), Hôpital d'Instruction des Armées de Percy, Favaro (18)] ou encore à la sous-traitance de certaines activités (9), jusqu'à des démarches beaucoup plus globales, reposant sur l'analyse de l'ensemble des flux physiques. Ainsi, le CHU de Montpellier a-t-il mis en place une plate-forme logistique qui centralise l'ensemble des flux physiques, depuis la papeterie ou les produits d'entretien jusqu'aux médicaments et dispositifs médicaux. Cette organisation est notamment couplée à une informatisation de la gestion des stocks, mais aussi de la gestion des commandes émanant des services de soins, à l'aide d'une identification par code barre des produits à commander. Un autre établissement peut être également cité en exemple : l'hôpital européen Georges Pompidou, qui a été conçu et organisé sur le plan architectural, en fonction de l'analyse des flux de patients (des urgences, des consultations,...), de la restauration, des examens de biologie,... (9 ou 19). Le CHRU de Tours a également mis en œuvre une démarche de réflexion autour de la logistique, pour aboutir à la création d'un logipôle, qui a pour vocation de regrouper sur un site unique l'ensemble des flux logistiques. Ce logipôle repose sur une informatisation pragmatique et opérationnelle, sur une contractualisation des rapports entre équipes logistiques et soignantes, et sur une intégration des unités de soins aux équipes de production dès la conception des prestations alimentaires (10).

Cela ne signifie pas pour autant que la gestion des flux de patients soit négligée, bien au contraire. Le patient étant au cœur de l'hôpital, ces préoccupations logistiques font l'objet d'améliorations probablement antérieures à celles portant sur les flux classiques. Toutefois, la logistique de service n'est pas toujours prise en compte comme une logistique à part entière ; elle relève plutôt d'améliorations fragmentées et ponctuelles.

Malgré les enjeux que représente l'amélioration de la démarche logistique, au travers d'une plus grande intégration intra et inter-organisationnelle, les limites à sa mise en œuvre effective ne doivent pas être négligées. Une première limite est liée au faible niveau général de maturité logistique au sein même des hôpitaux publics français, ce qui fera l'objet du paragraphe suivant. Les autres limites traitées ensuite sont avant tout organisationnelles et humaines.

\section{Typologie des pratiques logistiques des hôpitaux publics français}

\section{Une première limite à l'intégration de la logistique : le faible développement des établissements français}

La proposition d'une typologie des pratiques logistiques des hôpitaux publics français permet de mettre en évidence les disparités des niveaux de maturité logistique entre établissements. Cette typologie est le résultat d'une étude conduite en 1999, par le biais d'un questionnaire administré par voie postale auprès de cadres infirmiers de services chirurgicaux de 81 hôpitaux publics de type médecine-chirurgie-obstétrique. Elle a été mise en évidence par une ACM (analyse en composantes multiples), suivie d'une classification. 
Le design statistique a été appliqué en distinguant trois types de flux : les flux de patients, les flux de consommables provenant de la pharmacie et les flux provenant des services économiques. Ces derniers n'ont pu donner lieu à la proposition d'une typologie, les pratiques étant globalement très homogènes entre les différents établissements composant l'échantillon. En revanche ce résultat met en évidence le faible degré de développement des pratiques à ce niveau de l'organisation, les flux étant plutôt poussés, la répartition des tâches logistiques fragmentée à semi-intégrée et le style de coordination, pour une très large majorité de l'échantillon, de nature émergente, c'est-à-dire reposant sur des supports de communication peu sophistiqués (essentiellement papiers). Ce qui confirme en partie l'idée selon laquelle les hôpitaux ont une vision tronquée de la logistique. En revanche, pour les deux autres types de flux, les résultats sont plus mitigés.

Il est difficile de positionner les établissements dans une tendance précise quant au degré de tension des flux. On peut toutefois noter qu'environ $50 \%$ des répondants relèvent d'une gestion en flux poussés ou planifiés. Or, une inversion des flux serait bénéfique à divers titres. Les niveaux de stockage étant ajustés en fonction des consommations, les améliorations pourraient se traduire à la fois sur la quantité des marchandises stockées, mais aussi sur la qualité même du stock et éviter ainsi les risques de ruptures ou la péremption de certains médicaments.

L'intégration des fonctions logistiques dans les services de soins est pratiquée en partie pour environ un tiers des établissements interrogés. Seuls cinq hôpitaux ont mis en place une plate-forme regroupant les flux provenant de la pharmacie et des services économiques. La rareté des rapprochements existants entre ces deux services se justifie avant tout par des dispositions réglementaires imposant que le contrôle des médicaments soit réalisé par un diplômé de pharmacie. Toutefois, ces plate-formes ne sont pas forcément associées à une gestion partagée des stocks, qui sont présents dans les services de soins. Laisser le personnel des services logistiques réaliser l'ensemble des tâches logistiques aiderait notamment le personnel soignant à se centrer sur son cœur de métier, à savoir les soins aux patients. Ce point fera l'objet d'une réflexion plus approfondie.

Enfin pour ce qui est du style de coordination, on trouve des résultats comparables à ceux

Figure 1 - Typologie des pratiques logistiques pour les consommables venant de la pharmacie

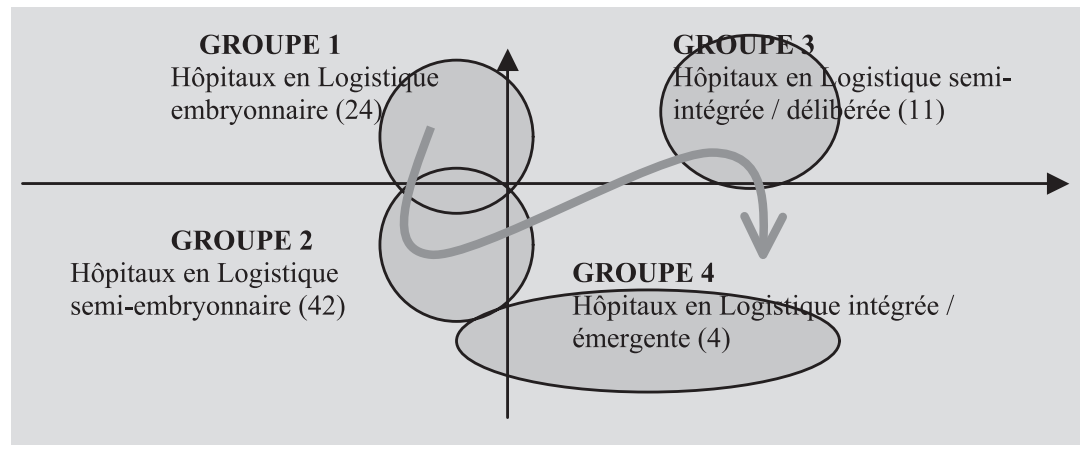

Figure 2 - Typologie des pratiques logistiques pour les trajectoires de patients

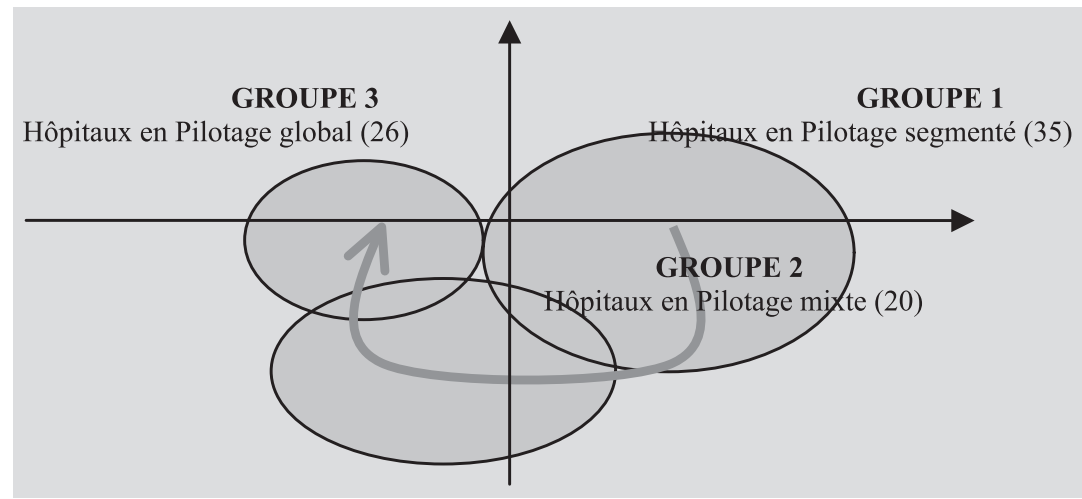

des flux de patients : $82 \%$ de l'échantillon utilisent des supports de communication peu ou pas sophistiqués, comme le papier ou les listings informatiques. Seuls $14,8 \%$ des établissements ont une gestion des stocks informatisée dans les services.

On note qu'un nombre important d'hôpitaux ont mis en place une démarche de préadmission (78\% de l'échantillon) et de prédiagnostic ( $88 \%$ de l'échantillon), ce qui permet aux patients d'éviter des temps d'attente parfois importants 4 .

En revanche, les résultats de l'enquête montrent que peu de services disposent d'un système permettant d'informer le bureau des entrées du nombre d'arrivées possibles. Or, un tel dispositif permettrait de faire remonter l'information pour tirer le flux, et, par exemple, de faire varier la capacité d'accueil du bureau des entrées (nombre de personnels, nombre de guichets etc.) en fonction du nombre de patients attendus [c'est une pratique de Yield Management].

L'intégration des services logistiques de type médico-techniques, que sont par exemple les laboratoires ou l'imagerie médicale, n'est pas pratiquée systématiquement. Par exemple :
4 - Il faut toutefois relativiser ce résultat, notamment concernant les prédiagnostics, l'activité des services étudiés étant chirurgicale, donc en plus grande partie programmée. 
Matrice des stades de développement logistique

\begin{tabular}{|c|c|c|c|c|}
\hline & \multicolumn{3}{|c|}{$\begin{array}{l}\text { STADES LOGISTIQUES / } \\
\text { TRAJECTOIRES DE PATIENTS }\end{array}$} \\
\hline & & $N^{\circ} 1$ & $\mathrm{~N}^{\circ} 2$ & $\mathrm{~N}^{\circ} 3$ \\
\hline \multirow{4}{*}{$\begin{array}{c}\text { STADES } \\
\text { LOGISTIQUES / } \\
\text { CONSOMMABLES } \\
\text { VENANT DE LA } \\
\text { PHARMACIE }\end{array}$} & $N^{\circ} 1$ & $\begin{array}{c}11 \\
\text { établissements } \\
\end{array}$ & 9 établissements & 4 établissements \\
\hline & $N^{\circ} 2$ & $\begin{array}{c}18 \\
\text { établissements }\end{array}$ & 8 établissements & $\begin{array}{c}16 \\
\text { établissements }\end{array}$ \\
\hline & $\mathrm{N}^{\circ} 3$ & 4 établissements & 3 établissements & 4 établissements \\
\hline & $N^{\circ} 4$ & 2 établissements & & 2 établissements \\
\hline
\end{tabular}

Note : le stade 1 représente le stade le moins développé, et les stades 3 ou 4, les stades les plus développés organisations françaises de santé en la matière. Cela reflète au contraire un espoir considérable pour l'évolution des organisations de santé : ces dernières ne se précipitent pas pour s'imiter les unes les autres, et pour adopter des solutions " clé en main » sans conduire auparavant une réflexion importante visant à analyser les spécificités du contexte.

Les limites à la mise en œuvre d'une approche intégrée de la logistique ne doivent pas être perçues comme des barrières au changement, mais au contraire comme des conditions de réussites à ce changement. Citons deux limites afin d'illustrer notre propos : les logiques professionnelles et les logiques organisationnelles.

\section{Les limites organisationnelles et humaines à une approche intégrée de la logistique}

nous incitent à émettre le postulat suivant : activités logistiques devraient être gérées par des personnes extérieures aux services de soins pour une meilleure efficacité [Chase (20)].

Le style de coordination est plutôt de nature émergente. Bien qu'un nombre important d'établissements cherchent à anticiper les déplacements des patients (63\% prennent rendez-vous au service d'imagerie médicale dès que l'hospitalisation est programmée), les supports utilisés pour communiquer entre services de soins et services médico-techniques sont peu informatisés (dans $20 \%$ des cas pour les prises de rendez-vous; dans $27 \%$ des cas pour la transmission des informations administratives des patients).

Si l'on croise les deux typologies obtenues, on observe une vision globale et hétérogène de la logistique pratiquée dans les établissements publics hospitaliers français.

Seuls 6 hôpitaux (gris foncé) parviennent à cumuler des stades de développement logistique élevés à la fois pour la logistique traditionnelle et la logistique de service. 20 établissements (gris clair) se situent à un stade élevé au niveau de la logistique de service, alors qu'ils témoignent d'un faible développement pour la logistique traditionnelle. L'inverse, un stade de développement élevé pour la logistique traditionnelle avec un faible niveau pour la logistique de service, est plus rare : 9 hôpitaux (diagonales) sont concernés.

Ces résultats mettent en évidence des disparités dans le niveau de maturité logistique entre les établissements hospitaliers. On pourrait le percevoir comme un retard important des
L'hôpital est un système multi-organisationnel composé en réalité de trois organisations : administrative, médicale et para-médicale, relevant respectivement d'un fonctionnement entrepreneurial, professionnel et mécaniste, en références aux travaux de Mintzberg [Cremadez (21), Guerrero (22) et Sampieri (3)]. Plus récemment, Glouberman et Mintzberg (23) ont mis en évidence la concomitance de quatre mondes fortement différenciés : communauté (les administrateurs), contrôle (les gestionnaires), traitement (les médecins) et soins (les infirmières).

Cette hétérogénéité organisationnelle a de nombreuses conséquences sur le fonctionnement des hôpitaux et plus particulièrement sur la gestion des flux. Ainsi la logistique n'est-elle pas gérée de façon uniforme, la partie « traditionnelle » (linge, restauration...) étant du ressort de l'administration et la partie «médico-technique » (imagerie médicale...) du centre opérationnel (composé des organisations médicale et para-médicale). Landry et Beaulieu (13) évoquent un «fractionnement des activités logistiques en une multitude d'acteurs ».

De plus, ces spécificités engendrent des dysfonctionnements dans le pilotage des flux. Le corps infirmier est chargé, sur ordre des médecins de façon formelle (ordonnances) ou de manière relativement autonome, de mobiliser l'ensemble des ressources nécessaires au fonctionnement du centre opérationnel. En effet, ainsi que le notent Glouberman et Mintzberg (23) « (...) les infirmières font fonctionner les unités de soins, cherchant à 
coordonner les flux de travail complexes ». Cependant, la faible considération qu'ont les médecins de l'aspect organisationnel de la mobilisation des ressources [Cremadez (24)] peut conduire le corps infirmier à « relâcher » la rigueur mise en œuvre pour gérer les activités logistiques. Cela se traduit par un recours abusif à l'ajustement comme mécanisme de coordination avec les services logistiques (procédures d'urgence), ou encore par un sur-stockage des produits, ces derniers constituant une marge de sécurité pour les acteurs et permettant une réduction des interdépendances vis-à-vis des services logistiques. L'émergence de ces pratiques autonomes, que l'on peut traduire en termes de slack organisationnel (au sens de Cyert et March) ou de zones d'incertitudes organisationnelles (au sens de Crozier et Friedberg), constitue une première limite à l'amélioration de la logistique hospitalière. Elle est d'autant plus importante qu'elle concerne une activité de service portant sur une cible humaine [Sampieri (25)] : la relation de front office qu'entretiennent les soignants avec les patients est un grand facteur d'incertitudes, la « cible étant intrinsèquement hétérogène ». Chaque patient revendique le droit d'être considéré comme une exception, ce qui influence fortement les soignants dans leur comportement, y compris dans les tâches relevant du back office, dont font partie les activités logistiques.

Ces éléments reflètent, en outre, la difficulté à intégrer la logistique dans les services de soins, notamment en déléguant la plupart des activités logistiques au personnel « purement » logistique : les différences culturelles entre ces deux organisations risquent d'engendrer des conflits d'interface portant par exemple sur la qualité des livraisons, leur fréquence, le respect des quantités commandées,... Une des solutions envisagée peut être l'intégration de soignants au sein des services logistiques en tant que correspondants privilégiés des services de soins.

Une autre limite doit être prise en compte avant de mettre en œuvre toute démarche intégratrice de gestion des flux : l'indépendance des professionnels (au sens de Mintzberg) que sont les médecins. Glouberman et Mintzberg (23) vont jusqu'à les situer à l'extérieur de l'organisation, dans la mesure où ils ne se rapportent pas à la hiérarchie de l'hôpital.

Une étude portant sur les prescriptions hospitalières de ville [SAMPIERI et al. (26)] a pu mettre en évidence l'autonomie des médecins dans l'orientation du patient vers une struc- ture hospitalière ou une structure de ville pour la réalisation d'examens de diagnostic. Les professionnels prennent en compte un nombre varié de critères pour orienter les patients, qui peuvent être différents d'un médecin à l'autre selon, notamment, sa place dans la filière de soins. Aussi, serait-il illusoire de vouloir optimiser la trajectoire d'un patient au sein de l'hôpital, voir au sein du réseau ou de la filière, sans prendre en considération le fait que les médecins appartiennent à une corporation. Tout comme dans le canal logistique industriel, il faut accepter des arbitrages entre optimums locaux et optimums globaux, dans la limite des libertés individuelles et des sphères de pouvoirs, qu'exercent les médecins. Il ne faut pas perdre de vue le fait que les flux de médicaments sont déclenchés par les médecins, à partir d'une ordonnance. Ces professionnels doivent donc être associés à toute démarche de réflexion portant sur la logistique, et ce, malgré leurs réticences... Tout comme, d'ailleurs, il est impératif de susciter l'intérêt de la Direction de l'hôpital.

Deux autres limites peuvent notamment être mises en évidence.

L'intégration de la logistique ne peut être dissociée des technologies de l'information et de la communication [Colin (7)]. Or, les systèmes d'information sont encore insuffisamment développés en matière de logistique dans les hôpitaux français. De plus, malgré des investissements importants dans le domaine, les compétences générales en informatique sont encore insuffisantes : une forte formation est donc nécessaire pour accompagner les changements d'envergure que pourrait engendrer une nouvelle démarche de gestion des flux.

Enfin, il faut prendre en compte le fait que, ainsi que le souligne HALGAND (27) en évoquant l'hôpital "Une des propriétés structurelles de ce type d'organisations réside dans une inadaptation très accusée à toute forme de contrôle -par les processus ou les résultats-, autre que très globale ou assimilable à un auto-contrôle ». Selon Glouberman et Mintzberg (23), cet impossible contrôle s'explique par une différenciation très marquée et croissante (entre les quatre mondes évoqués précédemment) et par un manque d'intégration. Or, on peut difficilement mettre en œuvre une démarche logistique qui s'intègre à l'ensemble de l'organisation et qui responsabilise les services logistiques, sans mettre en place des systèmes de contrôle de type tableau de bord, par exemple. En effet, 
ces éléments sont indispensables non seulement pour s'assurer de l'efficacité d'un système, mais aussi pour motiver le personnel, qui se sait évalué.

\section{Conclusion}

Cet article contient deux objectifs, qui peuvent paraître contradictoires : exposer les mérites d'une démarche logistique qui s'intègre à l'ensemble des fonctions de l'hôpital et de ses partenaires, et mettre en garde les acteurs contre les nombreuses limites liées à cette même démarche. Il ne suffit pas de s'apercevoir que la gestion des flux représente un gisement de productivité conséquent pour les établissements hospitaliers pour pouvoir mettre en œuvre les solutions adéquates.

D'une part, parce que l'hôpital n'est pas une organisation comme les autres : au delà de la sphère publique dans laquelle il est contraint, il est traversé par des logiques professionnelles, parfois corporatistes, qui complexifient toute démarche « purement » gestionnaire. En outre, la participation de plus en plus active du patient dans le processus de réalisation de l'activité hospitalière accentue cette complexification.

D'autre part, parce que la logistique est une démarche, un processus, qui modifie profondément l'organisation. Elle fait appel à des technologies de l'information et de la communication qui transforment les pratiques de travail. Elle transcende les frontières de l'organisation en imposant un interfaçage des fonctions.

Elle apporte une vision transversale, or l'hôpital a souvent été présenté, et l'est encore, comme une organisation où les cloisonnements sont particulièrement importants.

Dans ce contexte, il semble particulièrement intéressant de convaincre l'ensemble des acteurs hospitaliers, au-delà des logisticiens et des pharmaciens, de l'enjeu d'une telle démarche, non seulement pour dégager des ressources supplémentaires, humaines et financières, mais aussi, et surtout, pour améliorer encore les soins apportés aux patients. Une des difficultés majeures résidera sans doute dans la légitimation de l'acteur, qui sera responsable de la fonction logistique (et pas seulement manager des flux).

\section{Références bibliographiques}

(1) Landry, S. et M. Beaulieu (2000) (en collaboration avec T. Friel et C. R. Duguay) Etude internationale des meilleures pratiques de logistique hospitalière. Cahier de Recherche n00-05, ISSN : 1485-5496, Groupe Chaîne, HEC Montréal, $106 \mathrm{p}$.

(2) Mathe, H. (1997) Le service global-Innovations et stratégies internationales de développement dans les services. Maxima, Laurent Dumesnil Editions, Paris.

(3) Sampieri, N. (2000) Contribution à l'analyse de la logistique hospitalière : proposition d'une typologie des pratiques logistiques des hôpitaux publics français à partir d'une étude empirique. Thèse de Doctorat en Sciences de Gestion, Université de la Méditerranée, décembre.

(4) Colin, J. et G. Paché (1988) La logistique de distribution : l'avenir du marketing. Chotard et associés Editeurs.

(5) David, B.(1997) Les trois âges de la logistique : Mémoire d'une expérience pédagogique ; le cas du département « Transport Logistique » de l'IUT d'Aix-en-Provence. Communication à la 2ème conférence du Groupe de Recherche Aquitain et d'Analyse Logistique, Bordeaux. Le 27/02/1997.

(6) Heskett, J-L. (1977) Logistics, essential to strategy. Harvard Business Review. 55, $\mathrm{n}^{\circ} 6$.

(7) Colin, J. (2002) De la maîtrise des opérations logistiques au supply chain management. Gestion 2000. 1, 59-74.

(8) Caillet, R. (1995) Logistique et management hospitalier : l'expérience du CHRU de Lille. Logistique \& Management. 3, $\mathrm{n}^{\circ} 1$, 16-22.

(9) Cros, J-F. et J-P. Guillaume (1997) Dossier spécial : les enjeux de la logistique hospitalière. Logistiques Magazine. $\mathrm{n}^{\circ} 117$, 30-69.

(10) Moujart, M. (1999) Le CHRU de Tours à la découverte de la logistique hospitalière. Gestions Hospitalières. n³87, Cahiers Gestions Hospitalières, $\mathrm{n}^{\circ} 155$, 426-448.

(11) Actes du $3^{\mathrm{e}}$ forum AAQTE - APHAL du 22 et 23 novembre 2000 à Nancy (2000). Le Pharmacien Hospitalier. Supplément au $\mathrm{n}^{\circ} 142$.

(12) Bisch, F. (2000) Logistique et concept de Supply Chain dans le contexte de l'amélioration de la qualité des soins. Le Pharmacien Hospitalier. Supplément au n¹42, 27-28. 
(13) Landry, S. et M. Beaulieu (2002) La logistique hospitalière : un remède aux maux du secteur de la santé ? Gestion, vol. 26, n 4 , hiver, p.34-41.

(14) Techniques Hospitalières (1996). 608.

(15) Sampieri, N. (1999) Le développement potentiel de la logistique hospitalière : des enjeux pour l'amélioration de la qualité. Gestions Hospitalières, Cahier Gestions Hospitalières $n^{\circ} 155,449-453$.

(16) Hamel, G. et C.K. Prahalad (1990) « The Core Competence Of The Corporation "», Harvard Business Review, May-June, p.79-91.

(17) Gottsmann, J. (2000) La restructuration de la fonction " achats » d'un établissement hospitalier en Afrique noire - L'hôpital principal de Dakar. Gestions Hospitalières. Novembre, 695-699.

(18) Favaro, P. (2000) Analyse des consommations pharmaceutiques - Recherche de coûts standards par GHM «Brûlés ». Gestions Hospitalières. Novembre, 689-694.

(19) Ducasse, D. (1995) Comment une démarche logistique permet-elle d'atteindre les nouveaux objectifs économiques des Centres Hospitaliers ? L'exemple du projet de l'Hôpital Européen Georges Pompidou. Logistique \& Management. 3, 1, 3-9.

(20) Chase, R. B. (1981) The Customer Contact Approach To Services : Theoritical Bases And Practical Extensions. Operations Research. 29, 698-706.

(21) Crémadez, M. (1987) Les clés de l'évolution du monde hospitalier. Gestions Hospita- lières, Les cahiers de Gestions Hospitalières. $\mathrm{n}^{\circ} 266,285-306$.

(22) Guerrero, I. (1997) Une lecture de l'hôpital public fondée sur une triple approche de la structure. Journal d'Economie Médicale. 15, 5, 267-281.

(23) Glouberman, S. et H. Mintzberg (2002) Gérer les soins de santé et le traitement de la maladie. Gestion, vol.27, $\mathrm{n}^{\circ} 3$, automne, p.12-22.

(24) Crémadez, M. et F. Grateau (1992) Le management stratégique hospitalier. InterEditions, édition 1997.

(25) Sampieri, N. (2002) Différenciation et intégration du back office et du front office dans les activités de service - l'exemple de la logistique dans les établissements hospitaliers. La Revue des Sciences de Gestion, Direction et Gestion des Entreprises. n ${ }^{\circ} 195$, 31-40.

(26) Sampieri, N., T. Gullian et P. Mossé (2002) Les prescriptions « hospitalières de ville » - une pratique aux frontières. Journal d'Economie Médicale. 20, n¹, 19-32.

(27) Halgand, N. (1993) Pour une ingénierie contingente $d u$ contrôle - L'exemple de l'hôpital français. Thèse de Doctorat en Sciences de Gestion, Université de Montpellier 2, janvier.

(28) Sampieri-Teissier, N. (2002) Proposition d'une typologie des pratiques logistiques des hôpitaux publics français - Enseignements à partir d'une étude empirique. Logistique et Management, vol.10, $\mathrm{n}^{\circ} 1$, p.85-95. 